\title{
Review on glomus tumor of the temporal bone
}

\begin{abstract}
Glomus tumor is one of the common neoplasms of the temporal bone. This is a brief review on this neoplasm, its pathogenesis and treatment methods for that. This brief review tries to point to some important notes regarding glomus tumor of the temporal bone which knowing them would be important for the surgeons to approach such tumor more effectively.
\end{abstract}

Keywords: glomus tumor, temporal bone, surgical and radiation therapy

\author{
Volume 12 Issue 2 - 2020
}

\author{
Behzad Saberi \\ Medical Research, Iran \\ Correspondence: Behzad Saberi, Esfahan, Iran, \\ Tel 00989I3I263487, Email sab64b@yahoo.com
}

Received: January 23, 2020 | Published: April 22, 2020

\section{Mini review}

Glomus tumor is the second most common neoplasm of the temporal bone. The male to female ratio for such tumor would be one to five. The tumor is also known as Chemodectoma. About ten percent of the cases with such tumors are familial ones and the majority of the cases are sporadic. Glomus tumor is rarely functional and malignant in nature. Neuroendocrine system's chemoreceptor cells are the origin of glomus tumor. ${ }^{1,2}$ Mitochondrial DNA encoding for succinyl dehydrogenase subunit's C, D or B or mitochondrial complex II genetic defect, can be seen in the familial cases.

"Glasscock-Jackson" and "Fisch" are two systems for classification of glomus tumor. According to Fisch classification, the tumor classified into four types - A,B,C and D - and according to Glasscock-Jackson classification, the tumor would be classified into two subtypes which are Glomus jugulare and Glomus tympanicum and each can be sub classified into four types - I, II, III, IV. ${ }^{3}$

Glomus tumor may show itself with various symptoms like Aural fullness, facial weakness, otalgia, pulsatile tinnitus, dysphagia, hearing loss, hoarseness etc. Hypertension, palpitations and weight loss may be seen in functional tumors. Cranial nerve deficits and masses in the external auditory canal, neck or middle ear can be found in the physical examination of the patients with glomus tumor. CTscan, MRI, Angiography and Urine exam - to look for metanephrines or vanillyl mandelic acid - can be used for diagnosis. ${ }^{4}$

Surgery is the foundation for glomus tumor treatment. Glomus tympanicum can be surgically removed by employing tympanostomy approaches including anterior and posterior ones, transcanal and mastoidectomy surgical approaches.

Glomus jugulare can be surgically removed by employing infratemporal fossa approach. During approaching such tumors, enough care should be taken to preserve lower cranial nerves. Also great vessels proximal control in the sigmoid sinus and the neck, is necessary for surgical approach to such tumors. ${ }^{5,6}$ Facial nerve transposition for anterior tumor exposure may be done for large tumors either. In case the tumor would be very large in size and also during removal of the tumor from the temporal bone and the neck, if the amount of blood loss would be more than $3000 \mathrm{ml}$, surgical procedure 'staging' may become necessary to be done.

In the patients who do not want to do surgery or cannot tolerate that, radiation therapy can be used. For malignant tumors, lower doses of radiation will be used - about 15 Gy -. Further tumor growth can be prevented by using radiation therapy. Stereotactic radio surgery has a rate of about eighty percent in controlling the tumor, although employing stereotactic radio surgery may be concomitant with some risks including necrosis of the temporal bone, skull base osteoradionecrosis, causing injury to the cranial nerves and malignancy. ${ }^{7}$ It is important for the surgeon to have thorough knowledge about glomus tumor of the temporal bone, its etiology, clinical presentation and classification and also treatment strategies for such tumor to approach the relevant cases more effectively.

\section{Acknowledgments}

None.

\section{Conflicts of interest}

The author declares there are no conflicts of interest.

\section{Funding}

None.

\section{References}

1. Szymanska A, Szymanski M, Czekajska-Chehab E, et al. Diagnosis and management of multiple paragangliomas of the head and neck. Eur Arch Otorhinolaryngol. 2015;272(8):1991-1999.

2. van den Berg R. Imaging and management of head and neck paragangliomas. Eur Radiol. 2005;15(7):1310-1318.

3. Erickson D, Kudva YC, Ebersold MJ, et al. Benign paragangliomas: clinical presentation and treatment outcomes in 236 patients. $J$ Clin Endocrinol Metab. 2001;86(11):5210-5216.

4. van der Mey AG, Frijns JH, Cornelisse CJ, et al. Does intervention improve the natural course of glomus tumors? A series of 108 patients seen in a 32-year period. Ann Otol Rhinol Laryngol. 1992;101(8):635642.

5. Pellitteri PK, Rinaldo A, Myssiorek D, et al. Paragangliomas of the head and neck. Oral Oncol. 2004;40(6):563-575.

6. Taïeb D, Kaliski A, Boedeker CC, et al. Current approaches and recent developments in the management of head and neck paragangliomas. Endocr Rev. 2014;35(5):795-819.

7. Jackson CG, Glassock ME, Harris PF. Glomus tumors. Diagnosis, classification, and management of large lesions. Arch Otolaryngol. 1982;108(7):401-410. 